\title{
An ST2-dependent role of bone marrow-derived group 2 innate lymphoid cells in pulmonary fibrosis
}

\author{
Yuyue Zhao', Francina Gonzalez De Los Santos², Zhe Wu², Tianju Liu²*i] and Sem H Phan²* \\ Department of Respiratory Medicine, Peking Union Medical College Hospital, Chinese Academy of Medical Sciences and Peking Union Medical \\ College, Beijing, China \\ 2 Department of Pathology, University of Michigan Medical School, Ann Arbor, Michigan, USA
}

\begin{abstract}
*Correspondence to: Tianju Liu, MD, PhD, 4830 BSRB, Department of Pathology, University of Michigan Medical School, I09 Zina Pitcher Place, Ann Arbor, MI 48 109, USA. E-mail: tianliu@umich.edu;

Or Sem H Phan, MD, PhD, 4069 BSRB, Department of Pathology, University of Michigan Medical School, 109 Zina Pitcher Place, Ann Arbor, MI 48109, USA. E-mail: shphan@umich.edu
\end{abstract}

\begin{abstract}
Recent evidence supports that bone marrow (BM)-derived hematopoietic progenitor cells play an important role in lung injury and fibrosis. While these cells give rise to multiple cell types, the ST2 (II1 rl1)-expressing group 2 innate lymphoid cells (ILC2s) derived from BM progenitors have been implicated in tissue repair and remodeling, including in lung fibrosis. To further investigate the precise role of BM-derived ILC2s in the pathogenesis of fibrotic lung disease, their importance in the bleomycin-induced lung fibrosis model was evaluated by analyzing the effects of selective ST2 deficiency in the BM compartment. The results showed that while ST2-sufficient control mice exhibited activation of lung IL-33/ST2 signaling, ILC2 recruitment, IL-13 induction, and fibrosis, these responses were significantly diminished in ST2-deficient-BM chimera mice, with selective loss of ST2 expression only in the BM. This diminished response to bleomycin was similar to that seen in ST2 global knockout mice, suggesting the predominant importance of ST2 from the BM compartment. In wild-type mice, ILC2 recruitment to the lung was accompanied by a concomitant decrease in $\mathrm{ST}_{2}{ }^{+}$BM cells. ST2-deficient BM cells were unresponsive to IL-33-induced ILC2 maturation. Finally, lineage-negative wild-type, but not ST2-deficient BM cells from bleomycin-treated mice stimulated lung fibroblast type I collagen expression, which was associated with elevated TGF $\beta$ expression in the BM cells. Taken together, these findings suggested that the BM-derived ILC2s were recruited to fibrotic lung through the IL-33/ST2 pathway, and contributed to fibroblast activation to promote lung fibrosis. Copyright $\odot 2018$ Pathological Society of Great Britain and Ireland. Published by John Wiley \& Sons, Ltd.
\end{abstract}

Keywords: bone marrow; ILC2; IL-33; ST2; II1rl; fibrosis

Received I5 January 20 18; Revised 17 April 2018; Accepted 26 April 2018

No conflicts of interest were declared.

\section{Introduction}

Pulmonary fibrosis is a common end result of multiple insults to the lung [1]. Idiopathic pulmonary fibrosis (IPF) is the most common fibrotic interstitial lung disease of uncertain etiology with a poor prognosis [2]. A growing body of evidence suggests the importance of bone marrow (BM)-derived cells in animal model studies [3-6]. BM-derived lung hematopoietic progenitor cells rapidly migrate into the lung after injury, and subsequently contribute to fibrogenesis in a paracrine manner [4]. Moreover, BM CD11 $\mathrm{c}^{+}$ cell-derived amphiregulin promotes pulmonary fibrosis [7]. ST2-expressing group 2 innate lymphoid cells (ILC2s) represent another source of amphiregulin [8,9], and they have been implicated in fibrosis by studies analyzing the effects of deficiency of ST2 (Il1rl1) [10-12]. However, these studies cannot rule out the importance of ST2 in other (non-ILC2) cell types.
The ST2 ligand IL-33 is important for innate mucosal immunity in the lung and gut [13,14], and is also implicated in multiple pathogenic processes such as inflammation, tissue repair/fibrosis, and scleroderma $[10,15-17]$. It is expressed by endothelial and type II alveolar epithelial cells in the lung $[13,14,16]$. Its receptor ST2 is also widely expressed on multiple cell types including Th2 cells, mast cells, Th1 cells, regulatory $\mathrm{T}$ (Treg) cells, $\mathrm{CD}^{+} \mathrm{T}$ cells, natural killer (NK) cells, and group 2 innate lymphoid cells (ILC2s) [18]. ST2 (Illrll) pre-mRNA produces four isoforms through alternative splicing: ST2L, soluble ST2 (sST2), ST2V, and ST2LV [19]. ST2L is expressed on the membrane of hematopoietic cells [20], whereas sST2 is predominantly expressed by fibroblasts and epithelial cells functioning as a decoy receptor that exhibits anti-inflammatory properties [21]. ST2-expressing ILC2s represent important effector cells in the host response to infection, wound remodeling, allergic responses, fat biogenesis, and obesity [22-25]. 
ILC2s accumulate in tissue during type 2 inflammation, and function as a main source of cytokines such as IL-13, IL-4, IL-5, and amphiregulin [8,22,26-28], which are implicated in the process of tissue repair and fibrosis $[7,29,30]$. Both ST2 and IL-33 levels are induced in murine bleomycin (BLM)-induced pulmonary fibrosis and human IPF, and increased numbers of lung ILC2s are associated with fibrosis in the mouse [10,31,32]. Moreover, adoptive transfer of lung ILC2s enhances fibrosis in recipient mice, whereas anti-IL-33 antibodies or ST2 deficiency diminishes fibrosis [10]. These findings, along with association with alternative activation of macrophages (M2), suggest important roles for both ILC2s and M2 macrophages in fibrosis. However, that previous study relied on systemic antibody treatment or global ST2 knockout mice; thus, the diminished fibrosis cannot be attributed solely to ILC $2 s$ in view of the multiplicity of cell types that express ST2 and are IL-33 responsive. Furthermore, while the ILC2 adoptive transfer study indicates the ability of exogenous ILC2s to enhance fibrosis, it does not necessarily reveal a role for endogenous ILC2s in pathogenesis, nor does it give insight into the origin of lung ILC2s in pulmonary fibrosis.

In this study, we hypothesized the BM origin and paracrine role of ILC2 in BLM-induced lung fibrosis. To assess potential BM origin, we used ST2-deficient BM chimera mice lacking ST2 expression only in the $\mathrm{BM}$ and resulting in deficiency of BM-derived ILC2 because IL-33/ST2 signaling is essential for ILC2 differentiation/activation [24,33,34]. The results revealed that BLM-induced expansion of the lung ILC2 population and fibrosis was virtually abrogated in ST2-deficient BM chimera mice. Control lung ST2 expression and ILC2s were not affected by BM transplantation with $\mathrm{ST} 2-d e f i c i e n t$ BM. In vitro cell co-culture studies suggested paracrine regulation of myofibroblast differentiation by the BM-derived ILC2s, which if adequately amplified could serve as a potential mechanism by which ILC2s could promote fibrosis. These findings suggested that in response to a distal lung insult, ILC2s were recruited from the BM and activated/differentiated through IL-33/ST2 signaling, and in association with M2 macrophage differentiation contributed to fibroblast activation to promote the development of lung fibrosis in an ST2-dependent manner.

\section{Materials and methods}

\section{Mice and bleomycin model of pulmonary fibrosis}

C57BL/6 female mice (6-8 weeks of age) were obtained from The Jackson Laboratory (Bar Harbor, ME, USA). ST2 knockout (KO) mice on a C57BL/6 background were from Dr Stefan Wirtz (Friedrich Alexander Universität Erlangen, Erlangen, Germany). All mice were housed in the University Laboratory Animal Facility under animal protocols approved by the Institutional Animal Care and Use Committee at the University of
Michigan. The lung fibrosis model was induced by the endotracheal instillation of $2 \mathrm{U} / \mathrm{kg}$ BLM (Blenoxane; Mead Johnson, Princeton, NJ, USA) as described previously [3]. Control mice received the same volume of PBS only. ST2 KO BM chimera mice were generated by transplanting donor ST2 KO BM cells into recipient WT mice as described previously [3]. Mice receiving WT donor BM cells were used as controls [3]. The chimera mice were maintained on acidified water and autoclaved feed ad libitum for 6 weeks before use in the fibrosis model as described above.

\section{Collection of bronchoalveolar lavage fluid}

Bronchoalveolar lavage (BAL) was performed using $0.5 \mathrm{ml}$ of PBS and repeated three times for collection of BAL fluid. BAL fluid was then centrifuged at $500 \times g$ for 10 min to pellet cells. Total protein was measured using a Pierce BCA protein assay kit (Thermo Fisher Scientific, Rockford, IL, USA).

\section{Cell isolation}

Single cell suspensions of lung cells for flow cytometry and primary mouse lung fibroblasts were isolated and cultured as described previously $[3,35]$.

Lineage-negative $\left(\mathrm{Lin}^{-}\right) \mathrm{BM}$ cells were isolated from whole BM cell suspension using a mouse lineage cell cocktail kit (Miltenyi Biotec Inc, San Diego, CA, USA). Where indicated, isolated cells were treated with $30 \mathrm{ng} / \mathrm{ml}$ recombinant mouse IL-33 (R\&D Systems, Minneapolis, MN, USA) for $48 \mathrm{~h}$. For cell co-culture experiments, normal lung fibroblasts were plated $(8 \times$ 10 [4] cells per well) in the bottom chambers of 24-well Transwell plates with $0.4 \mu \mathrm{m}$-pore polycarbonate membrane inserts (Thermo Fisher Scientific) for $24 \mathrm{~h}$. The cells were starved in serum-free medium for another $24 \mathrm{~h}$, followed by the addition of $1.5 \times 10$ [6] $\mathrm{Lin}^{-}$ $\mathrm{BM}$ cells from WT or ST2 KO mice treated with PBS or BLM to the upper inserts. After an additional $24 \mathrm{~h}$ of incubation, the fibroblasts were collected for RNA isolation.

\section{Reverse transcription-PCR (RT-PCR) and ELISA}

RNA isolation and RT-PCR were performed as described previously [35]. Primers for assessing the mRNA levels for IL-33 (Il33), ST2L, type I collagen (Colla2), Acta2, TGF $\beta$ (Tgfb1), IL-13 (Il13), amphiregulin (Areg), TNF $\alpha$ (Tnf), Arg1, Nos2, and 18S rRNA were purchased from Thermo Fisher Scientific. Results were expressed as $2^{-\Delta \Delta C T}$ using the indicated control group as calibrator and 18S rRNA as reference 36 .

Soluble ST2 (sST2) levels in BAL fluid from control and BLM-treated mice and media from cell co-cultures were detected using an ST2 ELISA kit (R\&D Systems) in accordance with the manufacturer's instructions.

\section{Hydroxyproline and histological analysis}

Lung collagen content was determined by measuring the hydroxyproline content from lung homogenates as 
described previously [35]. The results were expressed as $\mu \mathrm{g}$ hydroxyproline per lung. The lung sections were stained with H\&E or Masson's trichrome for evaluation of histopathology.

\section{Flow cytometry}

Single cell suspensions from freshly digested lung tissue or BM were prepared as described above and analyzed by flow cytometry as described previously [37]. Dead cells were excluded from analysis using the Fixable Viability Dye eFluor ${ }^{\mathrm{TM}} 506$ (\#65-0866; dilution 1:1000; eBioscience, San Diego, CA, USA). Combinations of the following antibodies (and fluorophores) were used: anti-mouse ST2-PE-Cy7 (\#25-9335-80, clone: RMST2-2; dilution 1:100; eBioscience), IL-13-Alexa Fluor 488 (\#53-7133-82, clone: eBio13A; 1:100; eBioscience), ICOS-BV421 (\#564070, clone: 7E.17G9; 1:100; BD Biosciences, San José, CA, USA), Lineage-APC cocktail of anti-CD3e, CD11b, B220, TER-119, Ly-6C, Ly-6G (\#558074; 1:5; BD Biosciences), CD90.2-APC-Cy7 (\#105732, clone: 30-H12; 1:100; Biolegend, San Diego, CA, USA), CD45-Pacific Blue (\#103126, clone: 30-F11; 1:200; Biolegend), along with their respective isotype controls. The data were acquired using a NovoCyte flow cytometer and analyzed by NovoExpress software (Aeca Biosciences, Inc, San Diego, CA, USA). All other fluorescence conjugated-antibody controls except the target antibody were used for compensation.

\section{Statistical analysis}

All data are presented as mean values \pm SEM. Differences between any two groups were assessed for statistical significance by ANOVA followed by post hoc analysis using Scheffé's test. A $P$ value less than 0.05 was considered to be statistically significant.

\section{Results}

\section{Effects of selective BM ST2 deficiency on lung ILC2 induction and pulmonary fibrosis}

To study the involvement of the IL-33/ST2 signaling pathway and ILC2 in BLM-induced pulmonary fibrosis, we first confirmed that lung Il33 and ST2 mRNAs were highly induced at days 7 and 21 after BLM treatment (Figure 1A, B). The protein level of sST2 in BAL fluid was significantly induced at days 7 and 21 after BLM treatment (Figure 1C) but not in plasma (data not shown). Flow cytometry analysis revealed a significant increase in $\mathrm{Lin}^{-} \mathrm{CD} 45^{+} \mathrm{CD} 90.2^{+} \mathrm{ST}^{+}$ILC2s in BLM-injured lung at day 7 (Figure 1D), which was sustained at day 21 after BLM treatment (data not shown).

To exclude the contribution of ST2 in endogenous lung cell types and assess the specific importance of BM-derived ILC2s, we evaluated the in vivo effects of selective ST2 deficiency in BM progenitors using
ST2 KO BM chimera mice. Since ILC2s are developmentally derived from the BM $[34,38,39]$ with a dependence on IL-33/ST2 signaling for their differentiation/activation and recruitment to distal organs [24,33,34], this approach has been used as a means to assess the importance of BM-derived ILC2s. Indeed, flow cytometric analysis revealed that lung ILC2s were markedly reduced in $S T 2 \mathrm{KO}$ chimera mice due to a lack of ST2 in BM cells (Figure 2A, B), without significantly affecting differentiated $\left(\mathrm{Lin}^{+} / \mathrm{CD} 45^{+}\right.$ cells) myeloid cells $(10.2 \pm 1.9$ versus $11.09 \pm 2.23$ as $\%$ of total lung cells in control versus ST2 KO BM chimera mice, respectively) or CD11 $\mathrm{c}^{\mathrm{hi}} / \mathrm{MHCII}{ }^{\mathrm{hi}}$ cells $(0.83 \pm 0.17$ versus $1.15 \pm 0.01 \%$ of total lung cells in WT versus BM ST2 KO BM chimera mice, respectively) in the lungs of BLM-treated mice. Notably, the BLM-induced increase in lung ILC2s in WT mice was essentially abolished in ST2 KO BM chimera mice, which was accompanied by a significant reduction in BLM-induced $I l 13$ levels in ST2 KO BM chimera mice (Figure 2C). Thus, ST2 expression in BM progenitor cells was essential for recruitment/activation of lung ILC2s and IL-13 induction in pulmonary fibrosis.

Next, we investigated the impact of BM ST2 deficiency on pulmonary fibrosis. BLM induced extensive fibrosis in WT mouse lungs but it was attenuated and more circumscribed in the lungs of ST2 KO BM chimera mice (Figure 2D), which was accompanied by reduced deposition of collagen in ST2 KO BM chimera lungs, as shown by Masson's trichrome staining (supplementary material, Figure S1). This reduced fibrosis was confirmed quantitatively by a significant reduction in lung collagen content (Figure 2E) and Colla2 mRNA levels (Figure 2F). Significant inhibition of BLM-induced myofibroblast differentiation was also noted, as assessed by Acta 2 expression (Figure 2F). Additionally, the induction of Tnfa and Tgfbl was also inhibited in ST2 KO BM chimera lung after BLM treatment (Figure 2G). Finally, the BLM-induced increase in M2 marker Arg1 mRNA in WT controls was significantly reduced in ST2 KO BM chimera mice at day 7 but not at day 21 (Figure 2H). No significant effect was noted in the induction of the M1 marker Nos2 (data not shown). These findings together suggested that the BM ST2 deficiency prevented ILC2 recruitment/activation and early M2 polarization, which was accompanied by subsequent impairment of BLM-induced pulmonary fibrosis.

Given that the absence of ST2 expression was selectively confined to the BM compartment, this would suggest that the expansion in lung ILC2s in response to BLM-induced lung insult in WT mice might be due to IL-33/ST2-dependent recruitment of ILC2s or ILC2 precursors from the BM, as previously shown [34,38-40]. Flow cytometric analysis revealed that the number of $\mathrm{ST}_{2}{ }^{+}$cells was increased more than three-fold in the lung after BLM treatment (Figure 3A). In contrast, ST2 ${ }^{+}$ BM cells were significantly decreased after BLM treatment, which would be consistent with recruitment of these cells to the lung, resulting in their depletion in 
A

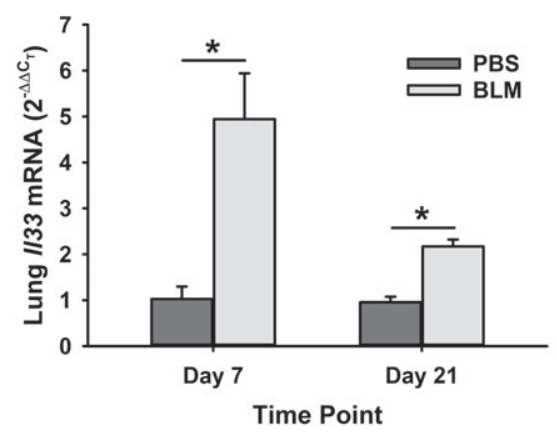

B

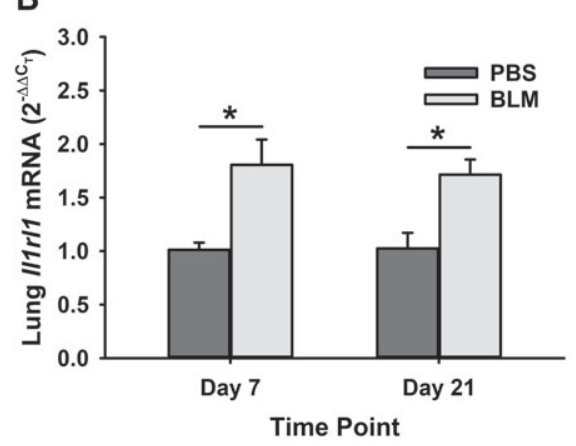

C

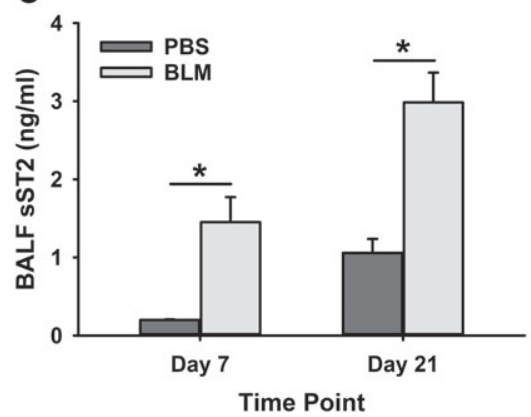

D
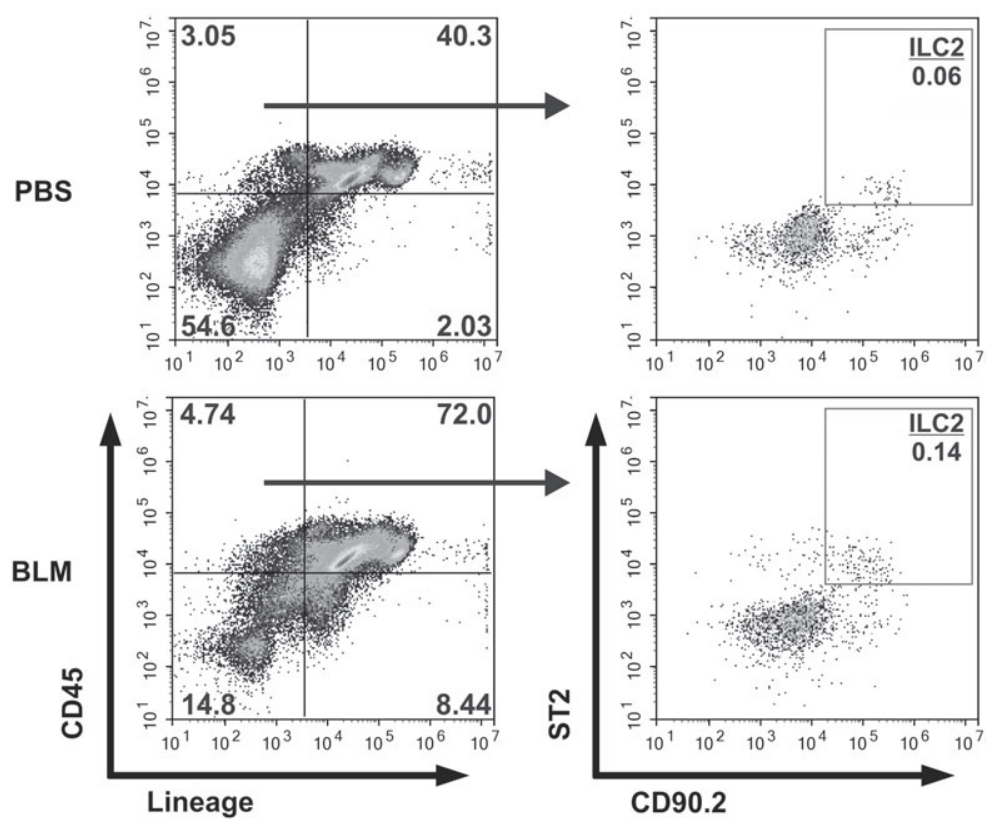

Figure 1. Effects of BLM on lung IL-33 and ST2 expression, and ILC2 cell number. Lung tissue RNA samples from BLM- or PBS-treated mice at days 7 and 21 after treatment were analyzed for $/ / 33$ (A) or ST2 (//1r/1) (B) mRNA levels by RT-qPCR. N=3 mice per group. In C, soluble ST2 protein levels in BAL fluid from BLM- or PBS-treated mice were quantified by ELISA $(N=3-8)$. In $D$, whole lung single cell suspensions were prepared 7 days after BLM treatment. ILC2s were gated as lineage-negative $\left(\mathrm{Lin}^{-}\right) \mathrm{CD} 45^{+} \mathrm{ST} 2^{+} \mathrm{CD} 90.2^{+}$. The cells were first gated on $\mathrm{Lin}^{-} \mathrm{CD} 45^{+}$and then analyzed for ST2 and CD90.2 expression for enumeration of ILC2s shown as a percentage of total cells. Data are shown as mean \pm SE. ${ }^{*} p<0.05$ between the two groups indicated in $\mathrm{A}-\mathrm{C}$. The experiments were independently repeated twice, and a representative data set is shown.

the originating BM. Since IL-33 is the cognate ligand for ST2, the response of ST2-deficient BM cells to IL-33 was evaluated to confirm its importance in ILC2 activation/differentiation. The results showed that while ILC2s were highly induced by IL-33 in WT Lin $^{-}$BM cells, ST2-deficient cells failed to respond (Figure 3B). Thus, BM ST2 ${ }^{+}$ILC2s or their progenitors were recruited to the lung in an IL-33/ST2-dependent manner and accounted for the expansion of lung ILC2s in BLM-induced pulmonary fibrosis.

\section{Comparative role of BM versus lung ST2 expression in BLM-induced pulmonary fibrosis}

To evaluate the relative importance of BM versus lung IL-33/ST2 signaling in vivo, BLM-induced lung fibrosis was also evaluated in WT and global ST2 KO mice.
Compared with WT mice, ST2 KO mice showed reduced BLM-induced lung fibrosis morphologically, with a smaller and less diffuse distribution of fibrotic lesions (Figure 4A), which was accompanied by a significant reduction in the BLM-induced increase of lung Colla2 (Figure 4B) and Acta2, a myofibroblast differentiation marker (Figure 4C). This reduction in BLM-induced pulmonary fibrosis in globally ST2-deficient mice was comparable to that seen in mice deficient in ST2 expression restricted to the BM compartment (Figure 2D, E).

Flow cytometric analysis of the lung cells revealed that the significant increase in lung ILC2s after BLM treatment also markedly reduced in global ST2 KO mice (Figure 5A), which was also comparable to that seen in mice with ST2 deficiency restricted to the BM (Figure 2B). This was accompanied by significantly 
A

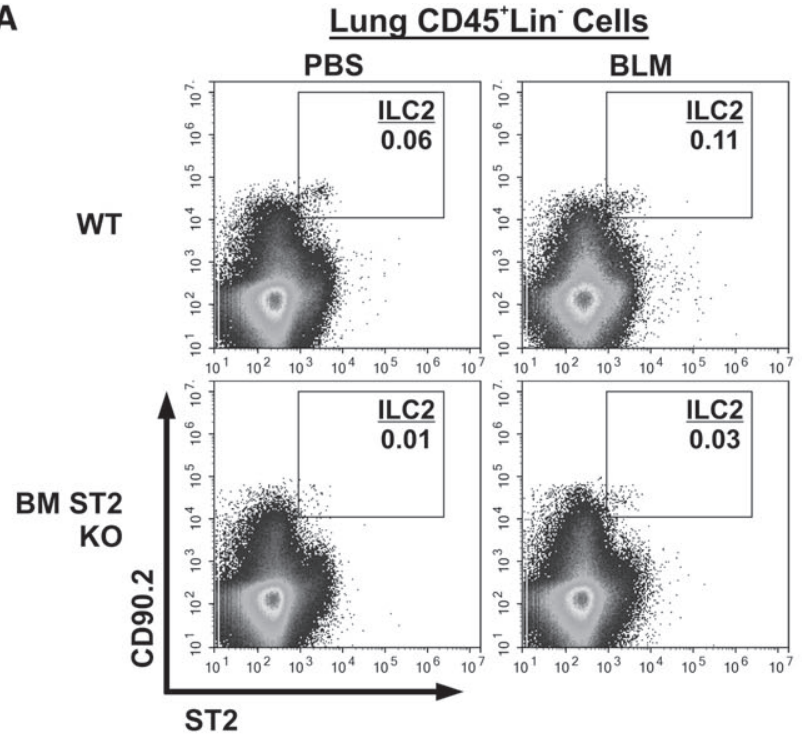

B

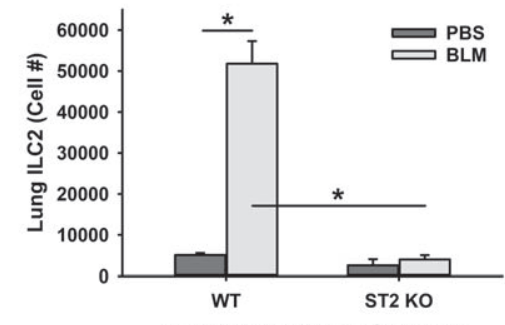

Bone Marrow Donor Genotype

C

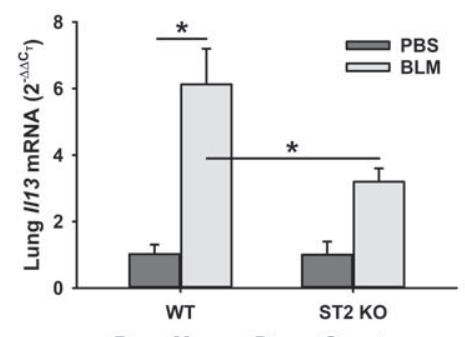

Bone Marrow Donor Genotype

D

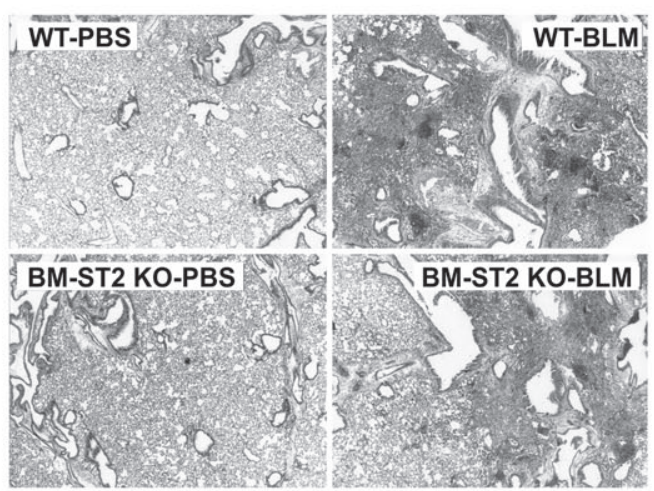

E

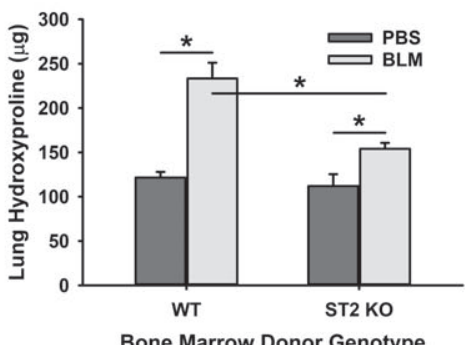

$\mathbf{F}$

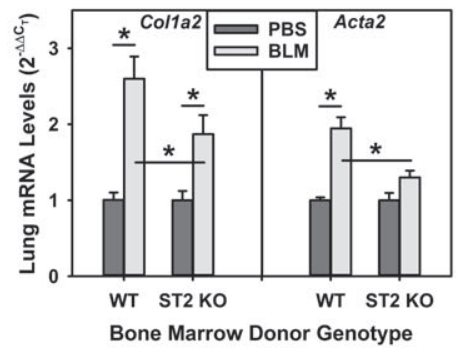

G

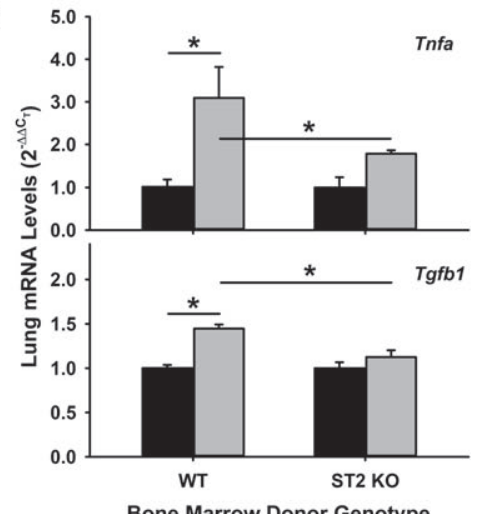

Bone Marrow Donor Genotype

H

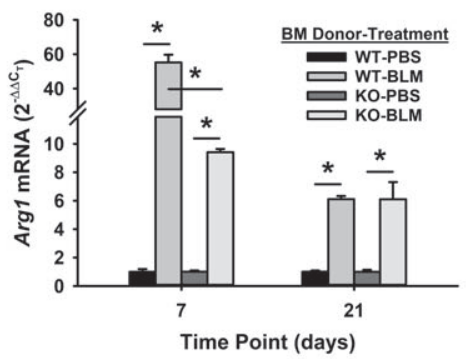

Figure 2. BLM-induced lung ILC2 recruitment and pulmonary fibrosis were abrogated in ST2 KO BM chimera mice. Lung cell suspensions from BLM- or PBS-treated WT (receiving WT BM) or ST2 KO BM chimera (receiving ST2 KO BM) mice at day 21 after treatment were analyzed for ILC2s as in Figure 1. A representative plot set is shown in A, and a summary from three separate experiments is shown in B. Data are shown as mean $\pm \mathrm{SE} . N=3 .{ }^{*} p<0.05$ between the two groups indicated. (C) Lung $/ / 13$ mRNA was analyzed from BLM- or PBS-treated ST2 KO BM chimera lung at day 21 after treatment. Data are shown as mean \pm SE. $N=3$ mice per group. ${ }^{*} p<0.05$ between the two groups indicated. (D) Representative lung tissue sections from WT or ST2 KO BM chimera mice treated as described in A were stained with HEtE (original magnification $\times 40$ ). (E) Lung tissue samples were evaluated for collagen content by hydroxyproline assay. (F) Lung tissue mRNA levels of type I collagen (Col1a2) and $\alpha$-SMA (Acta2). Tnfa and Tgfb1 (G), as well as the M2 macrophage marker Arg 1 (H), were analyzed by RT-qPCR. Data are shown as mean \pm SE. $N=5$ mice per group. ${ }^{*} p<0.05$ between the two groups indicated. 
A

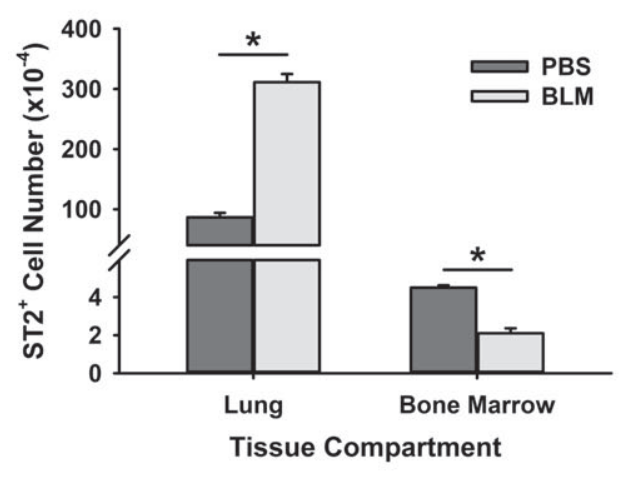

B

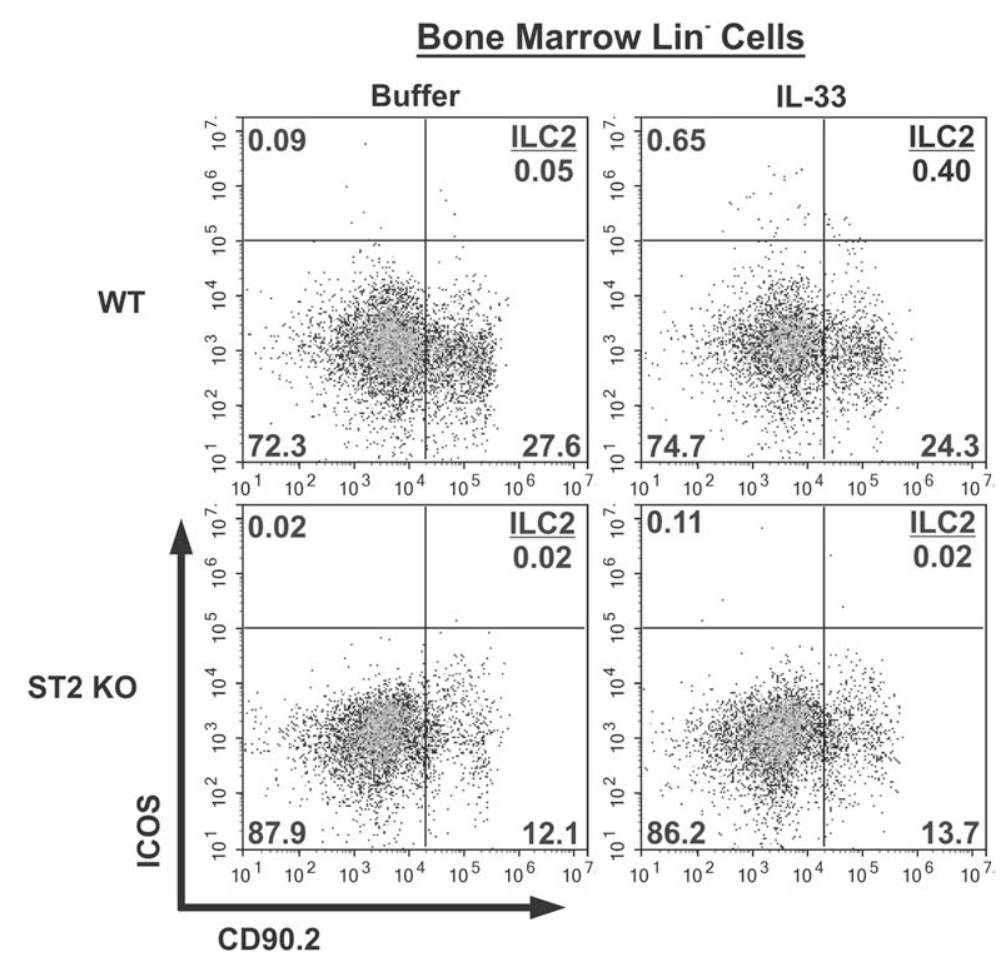

Figure 3. Effects of BLM on lung and BM ST2+ cells and ILC2 response to IL-33. (A) Lung and BM cells from BLM- or PBS-treated mice were analyzed for ST2 expression by flow cytometry, and the absolute numbers of ST2 $2^{+}$cells are shown. Data are shown as mean $\pm \mathrm{SE}$. $N=3$. ${ }^{*} p<0.05$ between the two groups indicated. (B) Lin- BM cells isolated from WT or ST2 KO mice were treated with $30 \mathrm{ng} / \mathrm{ml}$ IL-33 in vitro and then analyzed for ILC2 by flow cytometry. A representative scatter plot is shown. $N=3$ per group.

decreased numbers of IL-13-expressing ILC2s in BLM-treated ST2-deficient mice (Figure 5A). BLM induction of both lung IL-13 and amphiregulin expression was also reduced in ST2-deficient mice (Figure 5B, C). The reduction in lung $I l 13$ levels in global ST2 KO mice (Figure 5B) was also similar to that in mice lacking ST2 only in the BM (Figure 2C). These findings indicated that ST2 deficiency impaired the recruitment/activation and function of ILC2s in BLM-induced lung fibrosis, and that ST2 expression in the BM compartment was of predominant importance in the BLM-induced expansion of lung ILC2s and fibrosis.

\section{BM-derived ILC2s activated lung fibroblasts by increasing collagen I expression}

To elucidate the possible mechanism by which BM-derived ILC2s promoted lung fibrosis, we evaluated the effect of isolated BM-derived ILC2s on lung fibroblast activation. As only very few ILC2s could be isolated from mouse lungs, we used the more abundant $\mathrm{Lin}^{-} \mathrm{BM}$ cells to assess possible effects on lung fibroblast activation. These $\mathrm{Lin}^{-} \mathrm{BM}$ cells consisted of progenitor cells as well as ILC2s, and were used in co-cultures with normal lung fibroblasts. The results showed that $\mathrm{Lin}^{-} \mathrm{BM}$ cells isolated from BLM-treated WT mice caused a significant increase in Colla2 levels in lung fibroblasts, relative to $\mathrm{BM}$ cells from control PBS-treated mice (Figure 6A). However, BM cells from BLM-treated ST2 KO mice fibroblasts, compared with PBS-treated controls, failed to stimulate Colla2 levels. Interestingly, the $\mathrm{Lin}^{-} \mathrm{BM}$ cells isolated from BLM-treated WT mice expressed significantly higher levels of $T g f b 1$ relative to cells from WT PBS controls (Figure 6B), which was abrogated in BM cells from ST2 KO mice. This suggested that induction of TGF $\beta$ in BM progenitors and/or ILC2s could have caused the noted effects on fibroblast activation. This induction of TGF $\beta$ was likely due to BLM-induced IL-33, since it was dependent on ST2, and thus supported the identification of the $\mathrm{Lin}^{-} \mathrm{BM}$ cell as a likely ILC2 progenitor. Taken together, these findings would be consistent with BLM-induced lung IL-33 as an important signal for $\mathrm{BM}$ progenitor/ILC2 activation to enhance TGF $\beta$ expression leading to the activation of lung fibroblasts after BM-derived ILC2 recruitment to the lung.

\section{Discussion}

Innate lymphoid cells (ILCs) are recently discovered immune cells that reside at epithelial barriers such as the lung, skin, and gut, and contribute to immunity, inflammation, tissue homeostasis, and repair processes following exposure to allergens, pathogens or chemical irritants [41-43]. Three subsets of ILCs produce different type of cytokines. ILC2s produce type 2-associated cytokines, including IL-4, IL-5, and IL-13, as well as amphiregulin, thus playing important roles in parasite 
A

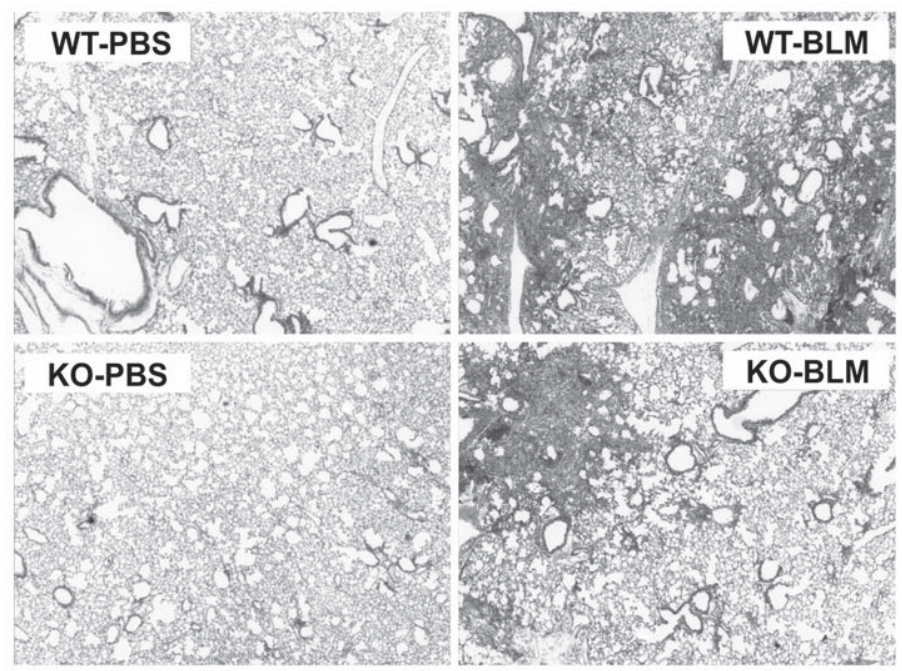

C

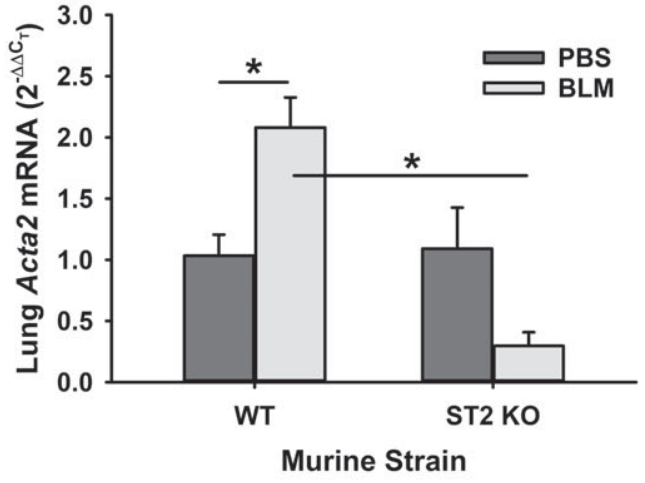

B

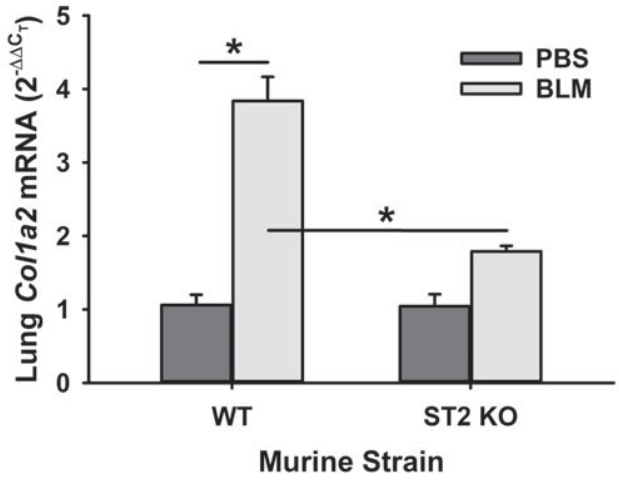

Figure 4. BLM-induced pulmonary fibrosis was attenuated in ST2 KO mice. WT or ST2 KO mice were treated with BLM or PBS endotracheally. Pulmonary fibrosis was evaluated at day 21 after treatment by histopathology ( $A$; representative tissue section shown; $\times 40$ objective magnification). Col1a2 (B) and Acta2 (C) mRNA levels. Data are shown as mean \pm SE. $n=3-5 .{ }^{*} p<0.05$ between the two groups indicated. The experiments were independently repeated twice, and a representative data set is shown.

A

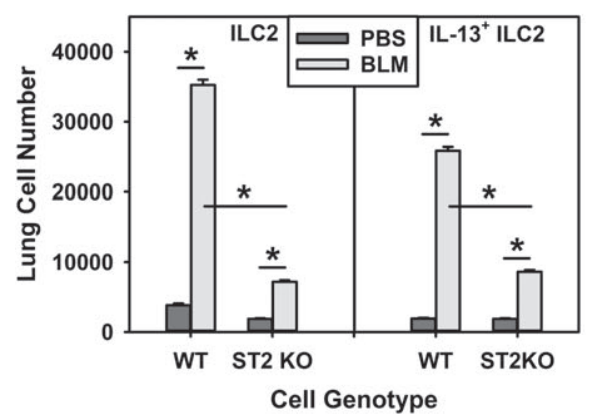

B

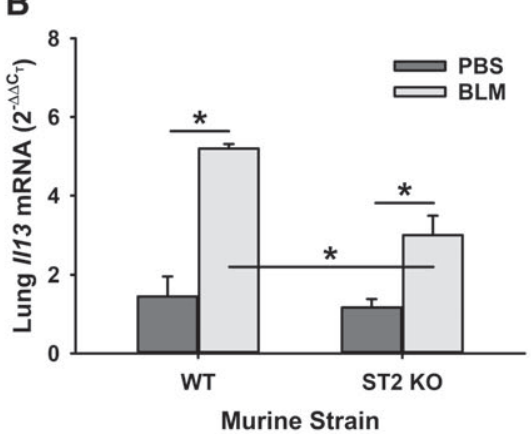

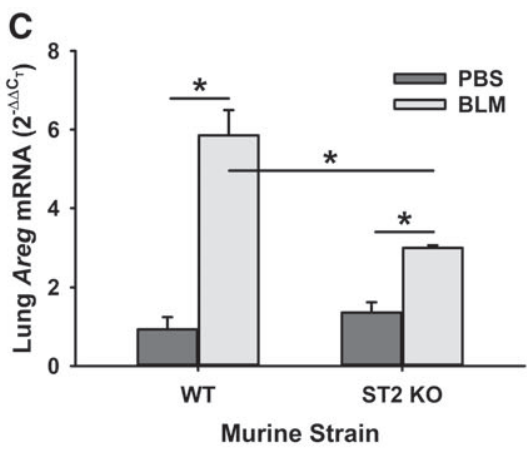

Figure 5. Lung ILC2 recruitment and activation were ablated in ST2 KO mice. Whole lung single cell suspension were prepared from day 7 PBS- or BLM-treated WT or ST2 KO lungs, and analyzed for ILC2s by flow cytometry and I/13 expression in ILC2s. (A) The absolute cell numbers per lung are shown for ILC2s and IL-13+ ILC2s. $N=3$ mice per group. Lung RNA samples from BLM- or PBS-treated mice were analyzed for IL-13 (B) or amphiregulin (Areg) (C) mRNA levels by RT-qPCR. $N=3$ mice per group. Data are shown as mean \pm SE. ${ }^{*} p<0.05$ between the two groups indicated.

immunity, allergic inflammation, and tissue homeostasis and fibrosis $[23,24,26,44,45]$. The ST2 ligand, IL-33, is a potent inducer of ILC2 activation $[8,16]$. The present study provided evidence that the IL-33/ST2 signaling pathway was induced in a model of lung injury and fibrosis through which the ILC2s were recruited from the BM and activated in the injured lung to promote fibrosis, perhaps by activating lung fibroblasts via TGF $\beta$.
IL-33 induction was also found in the BAL fluid in patients with IPF [46]. The elevated sST2 level in the BAL fluid in BLM-induced lung fibrosis was also consistent with activation of the IL-33/ST2 signaling pathway in lung fibrosis. The basis for this activation of ST2 signaling in pulmonary fibrosis may be due to the induction of IL-33 in injured epithelial cells serving as an alarm signal. Elevated sST2 in BAL fluid, but 

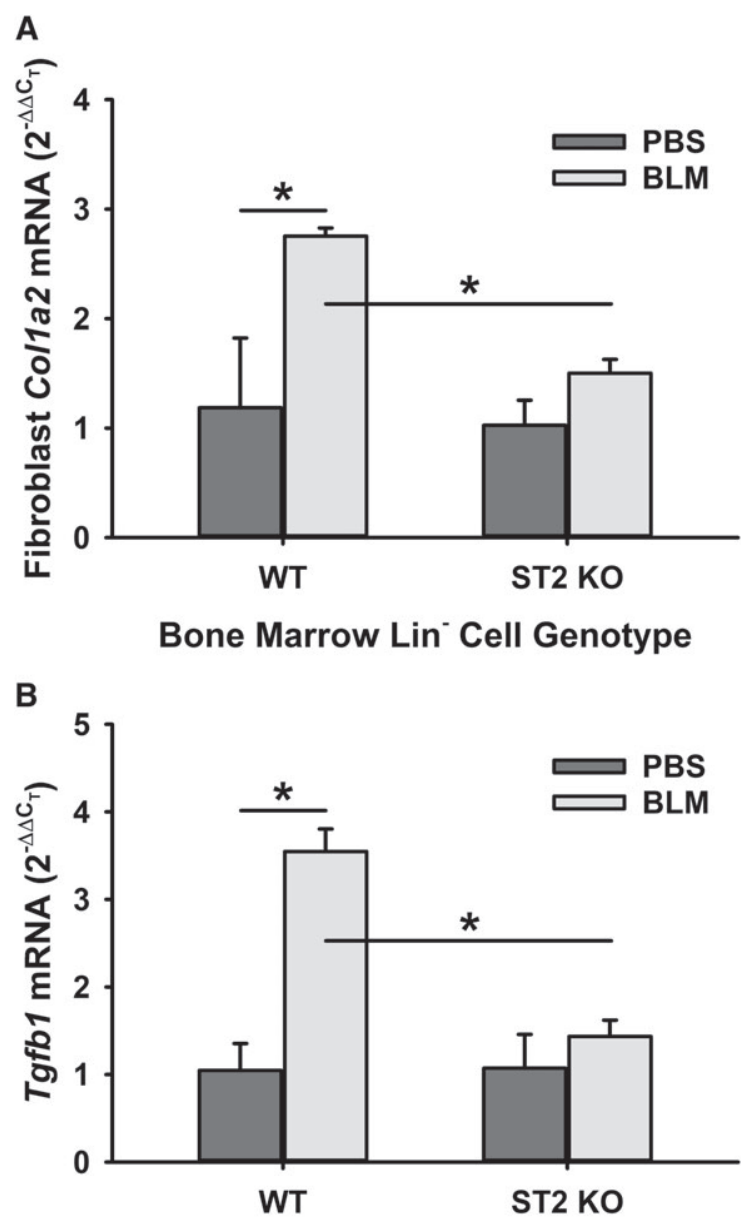

Bone Marrow Lin Cell Genotype

Figure 6. Lung fibroblasts were activated by BM-derived progenitor cells from BLM-treated mice. (A) Normal lung fibroblasts were co-cultured with Lin- BM cells isolated from BLM- or PBS-treated WT or ST2 KO mice 21 days after treatment. Fibroblast Col1a2 was analyzed by RT-qPCR. In B, similarly isolated Lin- BM cells from the same groups of mice were analyzed for Tgfb 1 mRNA by RT-qPCR. Data are shown as mean \pm SE. $N=5$ mice per group. ${ }^{*} p<0.05$ between the two groups indicated.

not in plasma, during lung fibrosis was sustained until the late stage of fibrosis at day 21. As an endogenous decoy receptor of IL-33, sST2 also antagonizes IL-33 and inhibits the IL-33/ST2L-induced Th2 response, and exogenous sST2 administration reduces BLM-induced fibrosis [47-49]. The significance of this induction of sST2 is unclear, given its paradoxical inhibitory effect on IL-33-ST2 signaling. It may be that the site of SST2 secretion occurs at a different location to where IL-33 is released from damaged cells. Further studies are needed to investigate the precise role of sST2.

The increase in IL-33-responsive ILC2s is not unique to animal models of pulmonary fibrosis as they are also increased in systemic sclerosis, correlating with the extent of fibrosis [50,51]. They are also present in the BAL samples and lung tissue of patients with IPF, wherein they are associated with upregulated expression of lung IL-33 and IL-25 [23,52]. The IL-33/ST2-induced increase in ILC2 number in BLM-induced lung fibrosis was ST2-dependent, as the ILC2 number and fibrosis were reduced in ST2 KO mice after BLM treatment. This is an expected finding given the dependence of ILC2 development on IL-33/ST2 signaling [24,33,34]. These findings are in agreement with another study where BLM-induced fibrosis was attenuated in global ST2 KO or by neutralizing IL-33 systemically through possible inhibition of M2 macrophage polarization [10]. Moreover, fibrosis is enhanced by adoptive transfer of ILC2s, although this does not indicate a requirement for endogenous ILC2s in fibrosis. Although BM ST2 deficiency also reduced M2 polarization, IL-33 alone cannot induce polarization [10]. While IL-13 alone can induce M2 polarization even in the absence of ST2, the addition of IL-33 magnifies this response $[10,53]$. This essential requirement for IL-13 to enable IL-33-induced polarization is due to the induction of ST2 by IL-13 [53]. Thus, it is likely that the induction of IL-13 in recruited ILC2s precedes and is essential for the subsequent enhanced M2 polarization by IL-33 [10,54]. IL-33-induced ILC2s are also an important source of IL-13 in liver fibrosis via the ST2-dependent signaling pathway [12], which would be consistent with the reduction in IL-13 expression in ST2-deficient mice in the BLM model of pulmonary fibrosis, thus impacting negatively on M2 polarization as noted.

However, studies using ST2 KO mice or antibodies to IL-33 cannot exclude impairment of IL-33/ST2 signaling in cells other than ILC2s as ST2 is expressed by diverse cell types [19]. To analyze more specifically the contribution of ILC2s in pulmonary fibrosis, we restricted the ST2 deficiency to the BM compartment by transplanting donor ST2 KO BM into WT recipients. Given that ILC2s originate developmentally from the BM and given their dependence on IL-33 (via ST2) for their development, this approach has been used as a means of depleting ILC2s [12,34,55]. Previously, ILC2s arising from BM progenitors were shown to migrate into lung in response to parasitic infection or allergic stimulation $[33,34,56]$. Using the $S T 2 \mathrm{KO}$ BM chimera mice in this study, we provided evidence that lung ILC2 development, recruitment, and activation were dependent on ST2 expression on the BM progenitor, and $\mathrm{Lin}^{-} \mathrm{BM}$ cells lacking ST2 expression failed to respond to IL-33 stimulation. Although this study did not completely exclude the potential impact of ST2 deficiency on other BM ST2-expressing cell types and their role(s) in fibrosis, there is evidence that ST2 deficiency has no significant impact on T-cell, mast cell, or lung macrophage development/function, or host defense against mycobacterial infection [10,57,58], consistent with a unique dependence of ILC2 differentiation on IL-33/ST2 signaling. Moreover, IL-33 overexpression in the BM has no significant impact on the number of eosinophils, neutrophils, basophils or M2 macrophages in the BM [59]. Other studies have used similar BM chimera strategies but using ROR $\alpha$-deficient BM donor cells to deplete ILC2s, which showed a similar lack of a significant effect on T cells [60]. 
Our results also revealed that $S T 2 \mathrm{KO} \mathrm{BM}$ chimera mice developed significantly decreased lung fibrosis and myofibroblast differentiation. These findings suggested that IL-33, as a signal, released from injured lung epithelial cells [16] initiated BM-derived ILC2 recruitment, development, and activation through the IL-33/ST2 signaling pathway with subsequent promotion of pulmonary fibrosis. Indeed, a recent study showed that ST2-dependent IL-33 signaling is a critical component in promoting egress of ILC2s from the BM in response to exogenous IL-33 administration, distal tissue depletion of ILC2s or fungal infection [34]. In our current study, the source of IL-33 was from the BLM-injured lung, and similar to this other recent study [34], deficiency of ST2 in the BM cells abrogated the response to the IL-33 from the lung. Other studies also provide support to the BM origin of ILC2s, especially under prolonged injury or disruption of homeostasis in tissues [34,40,61-64]. In our current study, the irradiation and BM transplantation procedure did not cause a significant change in the endogenous lung ILC2 population after stable engraftment was achieved (Figures 2B and 5C), suggesting that only the BLM insult-induced expansion in lung ILC2s was recruited from the BM, likely in response to the associated induction of lung IL-33 expression. Another recent study suggests the small intestine as an alternative site of origin for a subset of lung inflammatory ILC2s (iILC2s) [40]. Small intestine iILC2 precursors, not endogenous lung cells, migrate to the lung and give rise to proliferating iILC2s induced by IL-25 or helminth infection. However, it is noteworthy that the BM ST2-dependent recruited ILC2s in fibrotic lung with IL-33 induction in our study exhibited a $\mathrm{Lin}^{-} \mathrm{ST} 2^{+} \mathrm{CD} 90.2^{+}$phenotype, which differed from the IL-25 or helminth infection-induced lung Lin $^{-} \mathrm{KLRG}^{\mathrm{hi}} \mathrm{ST2}{ }^{-} \mathrm{CD} 90.2^{\text {lo }}$ iILC2s [40], suggesting that pulmonary fibrosis and acute helminth infection-induced inflammation may induce/recruit different ILC2 subsets potentially from different distal organ sites of origin. The mechanism of ILC2 recruitment appears to be IL-33/ST2-dependent in these and our current studies, but may be subject to regulation by additional agents, such as TGF $\beta$, which has been reported to enhance ILC2 chemoattractant activity [65].

The induction of IL-13 from recruited and activated ILC2s could account for their role in fibrogenesis $[12,29,30]$; however, direct effects of ILC2s on fibroblast activation have not been fully investigated. In our current study, an alternative paracrine role for ILC2s in fibrosis was suggested by the studies using co-culture of $\mathrm{Lin}^{-} \mathrm{BM}$ cells with lung fibroblasts. While ILC2s or their precursors comprise only a fraction of the $\mathrm{Lin}^{-} \mathrm{BM}$ cells used in this study, the dependence of the effects on ST2 expression makes it likely that any observed effects are due primarily to ILC2s and/or their precursors. The findings revealed induction of ST2-dependent TGF $\beta$ expression in BM cells in vivo in response to distal lung insult by BLM, perhaps caused by induction of lung IL-33 with consequent effects on ILC2 differentiation. This induction of TGF $\beta$ was associated with activation of the co-cultured lung fibroblasts, as manifested by increased collagen I expression. Such potential crosstalk between activated BM-derived ILC2s and lung fibroblasts via TGF $\beta$ could represent an additional novel mechanism by which ILC2s could promote lung fibrosis. While the relative number of lung ILC 2 s is small, it could have a disproportionately larger effect by amplification of paracrine effects in adjacent cells, including M2 polarization and potentially other BM-derived cell types known to play a role in fibrosis $[3,4,7,37]$. Moreover, TGF $\beta$ is known to be auto-inducible [66], such that the effect of the initial release by activated ILC2s can be amplified by auto-induction of greater levels of this cytokine in the initial target and other adjacent cells. Further studies are needed to fully explore the in vivo relevance of these in vitro findings and put them in the context of pulmonary fibrosis.

\section{Acknowledgements}

We thank Lisa R Riggs for excellent technical assistance in the tissue section preparation and the H\&E and Masson's trichrome staining. This study was supported by NIH grants HL052285 and HL112880 (to SHP) and a fellowship from The China Scholarship Council (to YZ).

\section{Author contributions statement}

YZ, TL, and SHP conceived experiments. YZ, FG, ZW, and TL carried out experiments and collected data. YZ, TL, and SHP analyzed data and wrote the manuscript. All authors were involved in writing the paper and had final approval of the submitted and published versions.

\section{References}

1. Wilson MS, Wynn TA. Pulmonary fibrosis: pathogenesis, etiology and regulation. Mucosal Immunol 2009; 2: 103-121.

2. Raghu G, Rochwerg B, Zhang Y, et al. An Official ATS/ERS/JRS/ALAT Clinical Practice Guideline: Treatment of Idiopathic Pulmonary Fibrosis. An Update of the 2011 Clinical Practice Guideline. Am J Respir Crit Care Med 2015; 192: e3-e19.

3. Hashimoto N, Jin H, Liu T, et al. Bone marrow-derived progenitor cells in pulmonary fibrosis. J Clin Invest 2004; 113: 243-252.

4. Nakashima $\mathrm{T}$, Liu $\mathrm{T}$, Yu $\mathrm{H}$, et al. Lung bone marrow-derived hematopoietic progenitor cells enhance pulmonary fibrosis. Am J Respir Crit Care Med 2013; 188: 976-984.

5. Moeller A, Gilpin SE, Ask K, et al. Circulating fibrocytes are an indicator of poor prognosis in idiopathic pulmonary fibrosis. Am J Respir Crit Care Med 2009; 179: 588-594.

6. Reilkoff RA, Bucala R, Herzog EL. Fibrocytes: emerging effector cells in chronic inflammation. Nat Rev Immuol 2011; 11: 427-435.

7. Ding L, Liu T, Wu Z, et al. Bone marrow CD11c+cell-derived amphiregulin promotes pulmonary fibrosis. J Immunol 2016; 197: 303-312.

8. Monticelli LA, Osborne LC, Noti M, et al. IL-33 promotes an innate immune pathway of intestinal tissue protection dependent on amphiregulin-EGFR interactions. Proc Natl Acad Sci U S A 2015; 112: $10762-10767$. 
9. Salimi M, Barlow JL, Saunders SP, et al. A role for IL-25 and IL-33-driven type-2 innate lymphoid cells in atopic dermatitis. $J$ Exp Med 2013; 210: 2939-2950.

10. Li D, Guabiraba R, Besnard AG, et al. IL-33 promotes ST2-dependent lung fibrosis by the induction of alternatively activated macrophages and innate lymphoid cells in mice. J Allergy Clin Immunol 2014; 134: 1422-1432.e11.

11. Rankin AL, Mumm JB, Murphy E, et al. IL-33 induces IL-13-dependent cutaneous fibrosis. J Immunol 2010; 184: $1526-1535$.

12. McHedlidze T, Waldner M, Zopf S, et al. Interleukin-33-dependent innate lymphoid cells mediate hepatic fibrosis. Immunity 2013; 39: $357-371$.

13. Schmitz J, Owyang A, Oldham E, et al. IL-33, an interleukin-1-like cytokine that signals via the IL-1 receptor-related protein ST2 and induces T helper type 2-associated cytokines. Immunity 2005; 23: 479-490.

14. Oboki K, Ohno T, Kajiwara N, et al. IL-33 is a crucial amplifier of innate rather than acquired immunity. Proc Natl Acad Sci U S A 2010; 107: $18581-18586$.

15. De la Fuente M, MacDonald TT, Hermoso MA. The IL-33/ST2 axis: role in health and disease. Cytokine Growth Factor Rev 2015; 26: $615-623$.

16. Yasuda K, Muto T, Kawagoe T, et al. Contribution of IL-33-activated type II innate lymphoid cells to pulmonary eosinophilia in intestinal nematode-infected mice. Proc Natl Acad Sci U S A 2012; 109: $3451-3456$.

17. Vettori S, Cuomo G, Iudici M, et al. Early systemic sclerosis: serum profiling of factors involved in endothelial, T-cell, and fibroblast interplay is marked by elevated interleukin-33 levels. J Clin Immunol 2014; 34: 663-668.

18. Liew FY, Girard JP, Turnquist HR. Interleukin-33 in health and disease. Nat Rev Immunol 2016; 16: 676-689.

19. Kakkar R, Lee RT. The IL-33/ST2 pathway: therapeutic target and novel biomarker. Nat Rev Drug Discov 2008; 7: 827-840.

20. Yanagisawa K, Takagi T, Tsukamoto T, et al. Presence of a novel primary response gene ST2L, encoding a product highly similar to the interleukin 1 receptor type 1. FEBS Lett 1993; 318: 83-87.

21. Takagi T, Yanagisawa K, Tsukamoto T, et al. Identification of the product of the murine ST2 gene. Biochim Biophys Acta 1993; 1178: 194-200.

22. Monticelli LA, Sonnenberg GF, Abt MC, et al. Innate lymphoid cells promote lung tissue homeostasis following acute influenza virus infection. Nat Immunol 2011; 12: 1045-1054.

23. Hams E, Armstrong ME, Barlow JL, et al. IL-25 and type 2 innate lymphoid cells induce pulmonary fibrosis. Proc Natl Acad Sci U S A 2014; 111: 367-372.

24. Halim TY, Steer CA, Matha L, et al. Group 2 innate lymphoid cells are critical for the initiation of adaptive $\mathrm{T}$ helper 2 cell-mediated allergic lung inflammation. Immunity 2014; 40: 425-435.

25. Hams E, Locksley RM, McKenzie AN, et al. Cutting edge: IL-25 elicits innate lymphoid type 2 and type II NKT cells that regulate obesity in mice. J Immunol 2013; 191: 5349-5353.

26. Price AE, Liang HE, Sullivan BM, et al. Systemically dispersed innate IL-13-expressing cells in type 2 immunity. Proc Natl Acad Sci U S A 2010; 107: 11489-11494.

27. Mjosberg JM, Trifari S, Crellin NK, et al. Human IL-25- and IL-33-responsive type 2 innate lymphoid cells are defined by expression of CRTH2 and CD161. Nat Immunol 2011; 12: 1055-1062.

28. Califano D, Furuya Y, Roberts S, et al. IFN- $\gamma$ increases susceptibility to influenza A infection through suppression of group II innate lymphoid cells. Mucosal Immunol 2018; 11: 209-219.

29. Doucet C, Brouty-Boye D, Pottin-Clemenceau C, et al. Interleukin (IL) 4 and IL-13 act on human lung fibroblasts. Implication in asthma. J Clin Invest 1998; 101: 2129-2139.
30. Lukacs NW, Hogaboam C, Chensue SW, et al. Type 1/type 2 cytokine paradigm and the progression of pulmonary fibrosis. Chest 2001; 120: 5S-8S.

31. Luzina IG, Kopach P, Lockatell V, et al. Interleukin-33 potentiates bleomycin-induced lung injury. Am J Respir Crit Care Med 2013; 49: $999-1008$.

32. $\mathrm{Xu} \mathrm{J}$, Zheng $\mathrm{J}$, Song $\mathrm{P}$, et al. IL-33/ST2 pathway in a bleomycin-induced pulmonary fibrosis model. Mol Med Rep 2016; 14: 1704-1708.

33. Chang YJ, Kim HY, Albacker LA, et al. Innate lymphoid cells mediate influenza-induced airway hyper-reactivity independently of adaptive immunity. Nat Immunol 2011; 12: 631-638.

34. Stier MT, Zhang J, Goleniewska K, et al. IL-33 promotes the egress of group 2 innate lymphoid cells from the bone marrow. J Exp Med 2018; 215: 263-281.

35. Liu T, Baek HA, Yu H, et al. FIZZ2/RELM- $\beta$ induction and role in pulmonary fibrosis. J Immunol 2011; 187: 450-461.

36. Livak KJ, Schmittgen TD. Analysis of relative gene expression data using real-time quantitative PCR and the 2(-Delta Delta C(T)) method. Methods 2001; 25: 402-408.

37. Ding L, Dolgachev V, Wu Z, et al. Essential role of stem cell factor-c-Kit signalling pathway in bleomycin-induced pulmonary fibrosis. J Pathol 2013; 230: 205-214.

38. Wong SH, Walker JA, Jolin HE, et al. Transcription factor ROR $\alpha$ is critical for nuocyte development. Nat Immunol 2012; 13: 229-236.

39. Yang Q, Saenz SA, Zlotoff DA, et al. Cutting edge: natural helper cells derive from lymphoid progenitors. J Immunol 2011; 187: 5505-5509.

40. Huang Y, Mao K, Chen X, et al. S1P-dependent interorgan trafficking of group 2 innate lymphoid cells supports host defense. Science 2018; 359: 114-119.

41. Artis D, Spits H. The biology of innate lymphoid cells. Nature 2015; 517: 293-301.

42. Spits H, Cupedo T. Innate lymphoid cells: emerging insights in development, lineage relationships, and function. Annu Rev Immunol 2012; 30: 647-675.

43. McKenzie ANJ, Spits H, Eberl G. Innate lymphoid cells in inflammation and immunity. Immunity 2014; 41: 366-374.

44. Molofsky AB, Van Gool F, Liang HE, et al. Interleukin-33 and interferon-gamma counter-regulate group 2 innate lymphoid cell activation during immune perturbation. Immunity 2015; 43: 161-174.

45. Rak GD, Osborne LC, Siracusa MC, et al. IL-33-dependent group 2 innate lymphoid cells promote cutaneous wound healing. J Invest Dermatol 2016; 136: 487-496.

46. Lee JU, Chang HS, Lee HJ, et al. Upregulation of interleukin-33 and thymic stromal lymphopoietin levels in the lungs of idiopathic pulmonary fibrosis. BMC Pulm Med 2017; 17: 39.

47. Pascual-Figal DA, Januzzi JL. The biology of ST2: the International ST2 Consensus Panel. Am J Cardiol 2015; 115: 3B-7B.

48. Seki K, Sanada S, Kudinova AY, et al. Interleukin-33 prevents apoptosis and improves survival after experimental myocardial infarction through ST2 signaling. Circ Heart Fail 2009; 2: 684-691.

49. Gao Q, Li Y, Pan X, et al. Lentivirus expressing soluble ST2 alleviates bleomycin-induced pulmonary fibrosis in mice. Int Immunopharmacol 2016; 30: 188-193.

50. Tajima S, Oshikawa K, Tominaga S, et al. The increase in serum soluble ST2 protein upon acute exacerbation of idiopathic pulmonary fibrosis. Chest 2003; 124: 1206-1214.

51. Xu J, Guardado J, Hoffman R, et al. IL33-mediated ILC2 activation and neutrophil IL5 production in the lung response after severe trauma: a reverse translation study from a human cohort to a mouse trauma model. PLoS Med 2017; 14: e1002365.

52. Wohlfahrt T, Usherenko S, Englbrecht M, et al. Type 2 innate lymphoid cell counts are increased in patients with systemic sclerosis 
and correlate with the extent of fibrosis. Ann Rheum Dis 2016; 75 : $623-626$.

53. Kurowska-Stolarska M, Stolarski B, Kewin P, et al. IL-33 amplifies the polarization of alternatively activated macrophages that contribute to airway inflammation. J Immunol 2009; 183: 6469-6477.

54. He R, Yin H, Yuan B, et al. IL-33 improves wound healing through enhanced M2 macrophage polarization in diabetic mice. $\mathrm{Mol}$ Immunol 2017; 90: 42-49.

55. Barlow JL, Peel S, Fox J, et al. IL-33 is more potent than IL-25 in provoking IL-13-producing nuocytes (type 2 innate lymphoid cells) and airway contraction. J Allergy Clin Immunol 2013; 132: 933-941.

56. Barlow JL, Bellosi A, Hardman CS, et al. Innate IL-13-producing nuocytes arise during allergic lung inflammation and contribute to airways hyperreactivity. J Allergy Clin Immunol 2012; 129: 191-198.e4.

57. Hoshino K, Kashiwamura S, Kuribayashi K, et al. The absence of interleukin 1 receptor-related T1/ST2 does not affect T helper cell type 2 development and its effector function. J Exp Med 1999; 190: 1541- 1548 .

58. Wieland CW, van der Windt GJ, Florquin S, et al. ST2 deficient mice display a normal host defense against pulmonary infection with Mycobacterium tuberculosis. Microbes Infect 2009; 11: 524-530.

59. Keller J, Catala-Lehnen $\mathrm{P}$, Wintges $\mathrm{K}$, et al. Transgenic over-expression of interleukin-33 in osteoblasts results in decreased osteoclastogenesis. Biochem Biophys Res Commun 2012; 417: 217-222.

60. Saranchova I, Han J, Zaman R, et al. Type 2 innate lymphocytes actuate immunity against tumours and limit cancer metastasis. Sci Rep 2018; 8: 2924.

61. de Kleer IM, Kool M, de Bruijn MJ, et al. Perinatal activation of the interleukin-33 pathway promotes type 2 immunity in the developing lung. Immunity 2016; 45: 1285-1298.

62. Gasteiger G, Fan X, Dikiy S, et al. Tissue residency of innate lymphoid cells in lymphoid and nonlymphoid organs. Science 2015; 350: 981-985.

63. Saluzzo S, Gorki AD, Rana BMJ, et al. First-breath-induced type 2 pathways shape the lung immune environment. Cell Rep 2017; 18: 1893-1905.

64. Steer CA, Martinez-Gonzalez I, Ghaedi M, et al. Group 2 innate lymphoid cell activation in the neonatal lung drives type 2 immunity and allergen sensitization. J Allergy Clin Immunol 2017; 140: 593-595.e3.

65. Denney L, Byrne AJ, Shea TJ, et al. Pulmonary epithelial cell-derived cytokine TGF- $\beta 1$ is a critical cofactor for enhanced innate lymphoid cell function. Immunity 2015; 43: 945-958.

66. Cutroneo KR, Phan SH. TGF- $\beta 1$-induced Smad 3 binding to the Smad 7 gene: knockout of Smad 7 gene transcription by sense phosphorothioate oligos, autoregulation, and effect on TGF- $\beta 1$ secretion: bleomycin acts through TGF- $\beta 1$. J Cell Biochem 2003; 89: 474-483.

\section{SUPPLEMENTARY MATERIAL ONLINE}

Figure S1. BLM-induced pulmonary fibrosis was abrogated in ST2 KO BM chimera mice 